\title{
Kindlin-2 regulates hepatic stellate cells activation and liver fibrogenesis
}

\author{
Jun Yu', Yinan $\mathrm{Hu}^{2}$, Yi Gao ${ }^{3}$, Qinghai $\mathrm{Li}^{2}$, Zhilin Zeng ${ }^{2,4}$, Yong $\mathrm{Li}^{2}$ and Huilong Chen ${ }^{2,4}$
}

\begin{abstract}
Liver fibrosis, the common response associated with chronic liver diseases, ultimately leads to cirrhosis, a major public health problem worldwide. Activation of hepatic stellate cells (HSCs) by transforming growth factor (TGF)- $\beta 1$ is a key step in liver fibrosis. Here we report that Kindlin-2 expression is elevated in the livers of mice with experimental liver fibrosis and also in the livers of patients with liver fibrosis. TGF- $\beta 1$ increases Kindlin- 2 expression in cultured HSCs in a p38 and ERK mitogen-activated protein kinase (MAPK)-dependent manner, partly. More importantly, Kindlin-2

deficiency significantly attenuated mouse liver fibrosis and HSC activation. Mechanistically, Kindlin-2 promotes TGF- $\beta$ signaling through upregulation of Smad2 and Smad3 phosphorylation. Our work demonstrates an important role for Kindlin-2 in liver fibrosis, and inhibiting Kindlin-2 in the livers may represent a novel strategy to treat liver fibrosis.
\end{abstract}

\section{Introduction}

Liver fibrosis is a tightly controlled wound healing response to virtually all forms of chronic liver injury due to viral infections, metabolic, and autoimmune diseases, which results in excessive accumulation of extracellular matrix $(E C M)$ and impaired normal liver function ${ }^{1}$. It is widely accepted that activated hepatic stellate cells (HSCs) play a pivotal role during development of liver fibrosis ${ }^{2}$. In response to liver injury, quiescent HSCs activate and differentiate into myofibroblast-like cells that are fibrogenic, contractile, and proliferative ${ }^{2,3}$. Transforming growth factor (TGF)- $\beta 1$ has been identified as the most potent mediator of HSC activation in liver fibrosis among several growth factors and cytokines ${ }^{4}$. TGF- $\beta 1$ signals through the canonical Smad signaling pathway involving TGF- $\beta$ receptor-mediated phosphorylation of Smad2 and Smad3 (p-Smad2/3), which form complexes with Smad4

\footnotetext{
Correspondence: Huilong Chen (huilong_chen@126.com)

${ }^{1}$ Department of Thoracic Surgery, Tongji Hospital of Tongji Medical College, Huazhong University of Science and Technology, Wuhan, China

${ }^{2}$ Department of Respiratory and Critical Care Medicine, Tongji Hospital, Tongji Medical College, Huazhong University of Science and Technology, Wuhan, China

Full list of author information is available at the end of the article. These authors contributed equally: Jun Yu, Yinan $\mathrm{Hu}$

Edited by N. Barlev
}

and translocate to the nucleus to regulate gene transcription $^{5,6}$. In addition to the Smad pathway, TGF- $\beta 1$ can also activate noncanonical Smad pathways, which including MAP kinase (MAPK) pathways, Rho-like GTPase signaling pathways, and phosphatidylinositol-3kinase (PI3K)/AKT pathways ${ }^{7}$. Both canonical and noncanonical pathways have been linked to HSC activation and liver fibrosis ${ }^{8}$.

Kindlins consist of three members, Kindlin-1, -2, and -3, which are a family of adapter proteins that mediate cell-cell and cell-matrix adhesions ${ }^{9-11}$. Kindlin-2, also known as Mig-2, encoded by FERMT2 gene, is widely expressed and evolutionarily conserved ${ }^{12}$. Kindlin-2 has emerged as a key regulator of integrin activation, which lead to actin remodeling, cell migration, and adhesion ${ }^{13,14}$. Global deficiency of Kindlin-2 in mice caused early embryonic lethality, suggesting that Kindlin-2 plays a vital role in development ${ }^{12}$. Furthermore, recent reports have demonstrated that Kindlin-2 participates in numerous biological and pathological processes, such as cell apoptosis, differentiation, survival, and carcinogenesis ${ }^{15-20}$. Recently, Kindlin-2 was also reported to play an essential role in fibrotic disorders. Wei and co-workers demonstrated that Kindlin-2 is highly expressed in human and

\section{(c) 2018 The Author(s).}

(c) (i) Open Access This article is licensed under a Creative Commons Attribution 4.0 International License, which permits use, sharing, adaptation, distribution and reproduction cc) in any medium or format, as long as you give appropriate credit to the original author(s) and the source, provide a link to the Creative Commons license, and indicate if changes were made. The images or other third party material in this article are included in the article's Creative Commons license, unless indicated otherwise in a credit line to the material. If material is not included in the article's Creative Commons license and your intended use is not permitted by statutory regulation or exceeds the permitted use, you will need to obtain permission directly from the copyright holder. To view a copy of this license, visit http://creativecommons.org/licenses/by/4.0/. 
mouse fibrotic kidney, and depletion of Kindlin-2 attenuates experimental renal fibrosis ${ }^{21}$; on the contrary, knockout of Kindlin-2 expression in cardiac myocyte or depletion of Kindlin-2 resulted in progressive cardiac fibrosis $^{22,23}$. Currently, the effect of Kindlin-2 in liver fibrosis remains unknown.

In the present study, we conducted a series of experiments to clarify the role of Kindlin-2 in the pathogenesis of liver fibrosis. We showed that Kindlin-2 is upregulated in human and mouse fibrotic livers, and this upregulation is mediated by TGF- $\beta 1$ through noncanonical Smad pathways in HSC, and then enhances HSC activation. Mechanistically, we demonstrated that Kindlin-2 mediates TGF- $\beta$ signaling through phosphorylation of $\operatorname{Smad} 2 /$ 3. Notably, depletion of Kindlin-2 dramatically inactivates the TGF- $\beta /$ Smad pathway and thereby prevents TGF- $\beta 1$ induced HSC activation and experimental liver fibrosis. Our study has uncovered an important role of Kindlin-2 in liver fibrosis progression and suggests that inhibition of Kindlin-2 may represent a novel therapeutic approach for the treatment of hepatic fibrosis.

\section{Results}

Kindlin-2 expression is upregulated in human and mouse liver fibrosis

To understand the role of Kindlin-2 in the progression of liver fibrosis, we initially assessed Kindlin-2 levels in human fibrotic livers. As shown in Fig. 1a, using immunofluorescence assay, we found that Kindlin-2 was highly expressed in human liver fibrosis. Double staining with $\alpha$ SMA showed that activated HSCs express significant high level of Kindlin-2. We then detected the Kindlin-2 expression in mouse fibrotic livers that were induced by intraperitoneal $\mathrm{CCl}_{4}$ injection, a well-studied mouse liver fibrosis model $^{24}$. We also observed elevated Kindlin-2 expression in experimental liver fibrosis. Immunofluorescence co-staining Kindlin-2 and $\alpha$-SMA highlighted a similar expression pattern in mouse as in human fibrotic livers with strongly increased Kindlin-2 levels in activated HSC (Fig. 1b). Moreover, western blot showed that the $\mathrm{CCl}_{4}$-induced mouse livers exhibited significant upregulation of Kindlin-2 compared with the control livers (Fig. 1C). The expression of tubulin was not changed upon $\mathrm{CCl}_{4}$ treatment. These data demonstrated that increased Kindlin-2 may serve as a critical marker of HSC activation.

\section{TGF- $\beta$ upregulates Kindlin-2 expression in HSC via p38 and ERK}

We next examined Kindlin-2 levels in LX-2 cells ${ }^{25}$, a well-established human HSC cell line upon TGF- $\beta$ treatment. As illustrated in Fig $2 \mathrm{a}, \mathrm{b}, \mathrm{TGF}-\beta 1$ increases Kindlin-2 protein levels in a time-dependent and dosedependent manner. In addition, elevated Kindlin-2 mRNA level was also observed in the whole RNA extracted from TGF- $\beta 1$-treated LX-2 cells (Fig. 2 c). To investigate how TGF- $\beta 1$ triggers Kindlin- 2 expression, TGF- $\beta 1$ signaling inhibitors were added before TGF- $\beta 1$ treatment. We observed that p38 MAPK inhibitor SB203580 and extracellular signal-regulated kinase (ERK) inhibitor U0126, but not c-Jun N-terminal kinase (JNK) inhibitor SP600125, blocked the increase effect of TGF- $\beta 1$ on Kindlin-2 expression in LX-2 cells (Fig. 2d, e). Taken together, we demonstrated that the TGF- $\beta 1$ triggers Kindlin-2 expression via p38 and ERK MAPK pathway.

\section{Kindlin-2 promotes TGF- $\beta$-induced HSC activation}

To investigate the functional effects of the increased expression of Kindlin-2, we used siRNA to knock down Kindlin-2 in LX-2 cells. Indeed, siRNA against Kindlin-2 successfully reduced the expression of Kindlin-2. Knockdown of Kindlin- 2 dramatically blocked TGF- $\beta$-induced Col1A1 and FN protein expressions (Fig. 3a). Kindlin-2 silencing also significantly suppressed the mRNA levels of Col1A1, FN, and $\alpha$-SMA, as showed in Fig. 3b-d. Furthermore, immunofluorescence staining demonstrated that knockdown of Kindlin-2 substantially attenuated the expressions of both FN and $\alpha$-SMA protein levels stimulated by TGF- $\beta 1$ (Fig. 3e). To further investigate the roles of Kindlin-2 in regulating HSC activation, we evaluated the overexpression of Kindlin-2 on LX-2 cells. As shown in Fig. 4a, enforced Kindlin-2 expression enhanced TGF- $\beta$-induced expressions of Col1A1 and FN. In addition, Kindlin-2 overexpression upregulated FN and $\alpha$ SMA protein levels triggered by TGF- $\beta 1$ that was confirmed by immunofluorescence assay (Fig. 4b).

\section{Kindlin- 2 contributes to the activation of TGF- $\beta / \mathrm{Smad}$ pathway}

TGF- $\beta /$ Smad signaling was demonstrated to participate in HSC activation and ECM production. Therefore, we determined that whether Kindlin-2 is required for the activation of TGF- $\beta /$ Smad pathway. As shown in Fig. $5 \mathrm{a}$, knockdown of Kindlin-2 with siRNA decreased phosphorylation of Smad2 and Smad3 protein levels in response to TGF- $\beta 1$ compared with control siRNA. In addition, immunofluorescence staining showed similar effect (Fig. 5b). Consistently, overexpression of Kindlin-2 indeed enhanced the TGF- $\beta 1$-induced phosphorylation of Smad2 and Smad3 (Fig. 5c), and these results were also observed using immunofluorescence assay (Fig. 5d). These results indicate that Kindlin-2 activates TGF- $\beta$ / Smad signaling through upregulation of Smad2 and Smad3 phosphorylation.

\section{Kindlin-2 deficiency reduces $\mathrm{CCl}_{4}$-induced liver fibrosis}

To investigate the role of Kindlin-2 in experimental liver fibrosis, the mouse model of $\mathrm{CCl}_{4}$-induced liver 


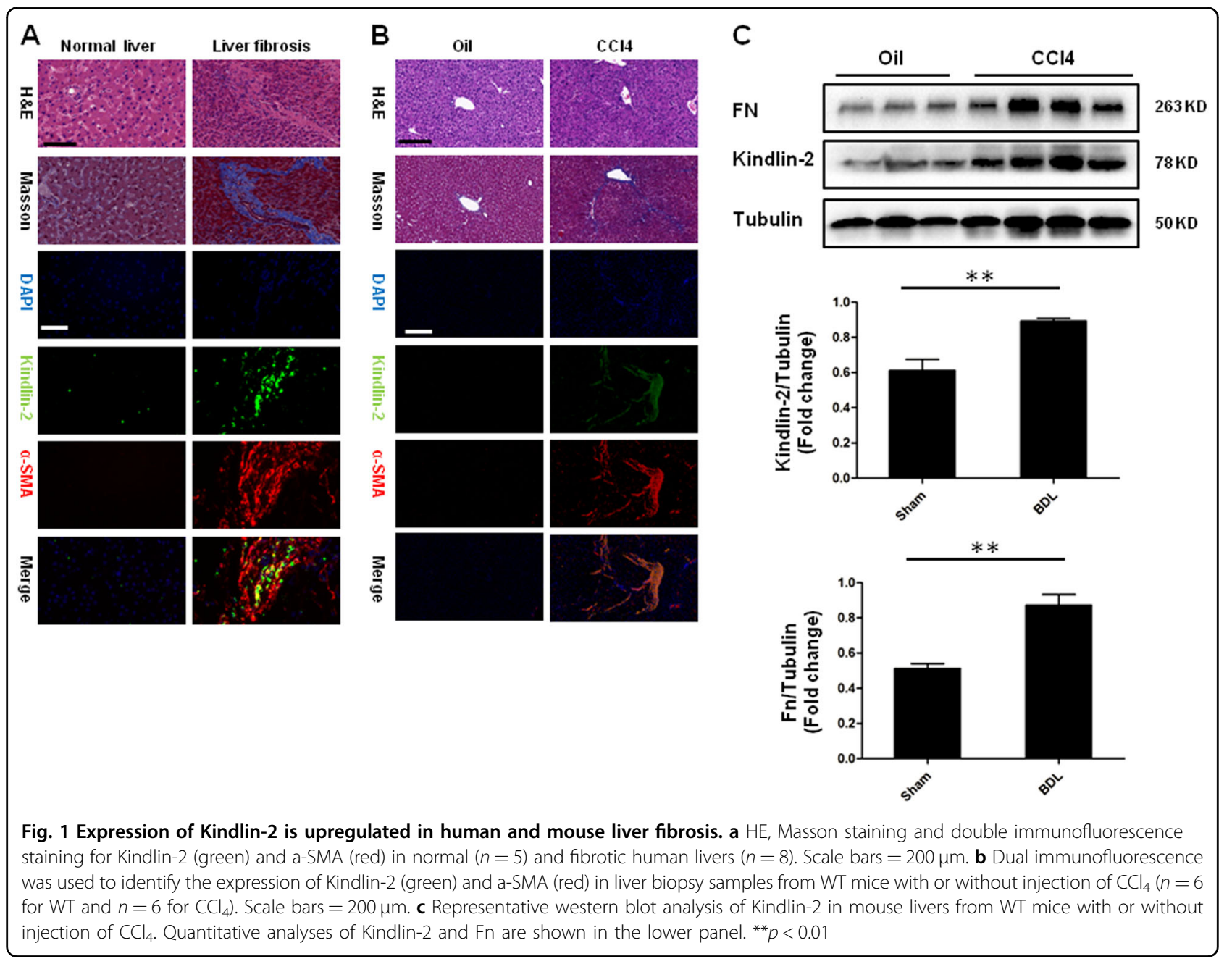

fibrosis was used. As shown in Fig. 6b, the expression of Kindlin-2 protein in Kindlin- $2^{+/-}$mouse livers was significantly reduced compared with control mice. The expression of beta-actin was not changed after Kindlin-2 depletion. After the treatment of mice with $\mathrm{CCl}_{4}$, we found that Kindlin-2 depletion significantly attenuated the liver collagen deposition illustrated by Sirius Red and Masson's trichrome staining (Fig. 6a). Moreover, we assessed the protein levels of $\alpha$-SMA in the liver by western blot analysis. $\alpha$-SMA protein levels were markedly diminished in the liver from $\mathrm{CCl}_{4}$-treated Kindlin- $2^{+/-}$mouse compared with WT mouse (Fig. 6c). Next, the mRNA expressions of Col1A1, Col1A2, and Col3A1 were significantly reduced in Kindlin- $2^{+/-}$mice livers compared with WT mouse after $\mathrm{CCl}_{4}$ injection (Fig. 6d). Collectively, these results demonstrated that Kindlin-2 deficiency significantly attenuated $\mathrm{CCl}_{4}$-induced liver fibrosis, suggesting that Kindlin-2 is a potential therapeutic target for liver fibrosis.

\section{Discussion}

In this article, we demonstrated for the first time that Kindlin-2 plays a key role in HSC activation and liver fibrosis. First, we showed that Kindlin-2 expression was markedly upregulated in mouse and human fibrotic livers. Second, TGF- $\beta 1$ significantly induced Kindlin- 2 expression in vitro, and knockdown of Kindlin-2 suppressed TGF- $\beta 1$-induced HSC activation. Third, Kindlin- 2 promoted TGF- $\beta$ signaling through activation of Smad2 and Smad3 phosphorylation. Finally, depletion of Kindlin-2 in vivo markedly attenuated $\mathrm{CCl}_{4}$-induced mouse liver fibrosis. All these data support our conclusion that Kindlin-2 functions as a positive regulator in liver fibrosis and may be a promising target for liver fibrosis treatment.

Recently, Lin et al. reported that Kindlin-2 expression is upregulated in hepatocellular carcinoma (HCC) and promotes HCC progression ${ }^{26}$; however, the function of Kindlin-2 in other liver diseases including liver fibrosis remains unknown. In the current study, we showed that Kindlin-2 expression was enhanced in livers from both 


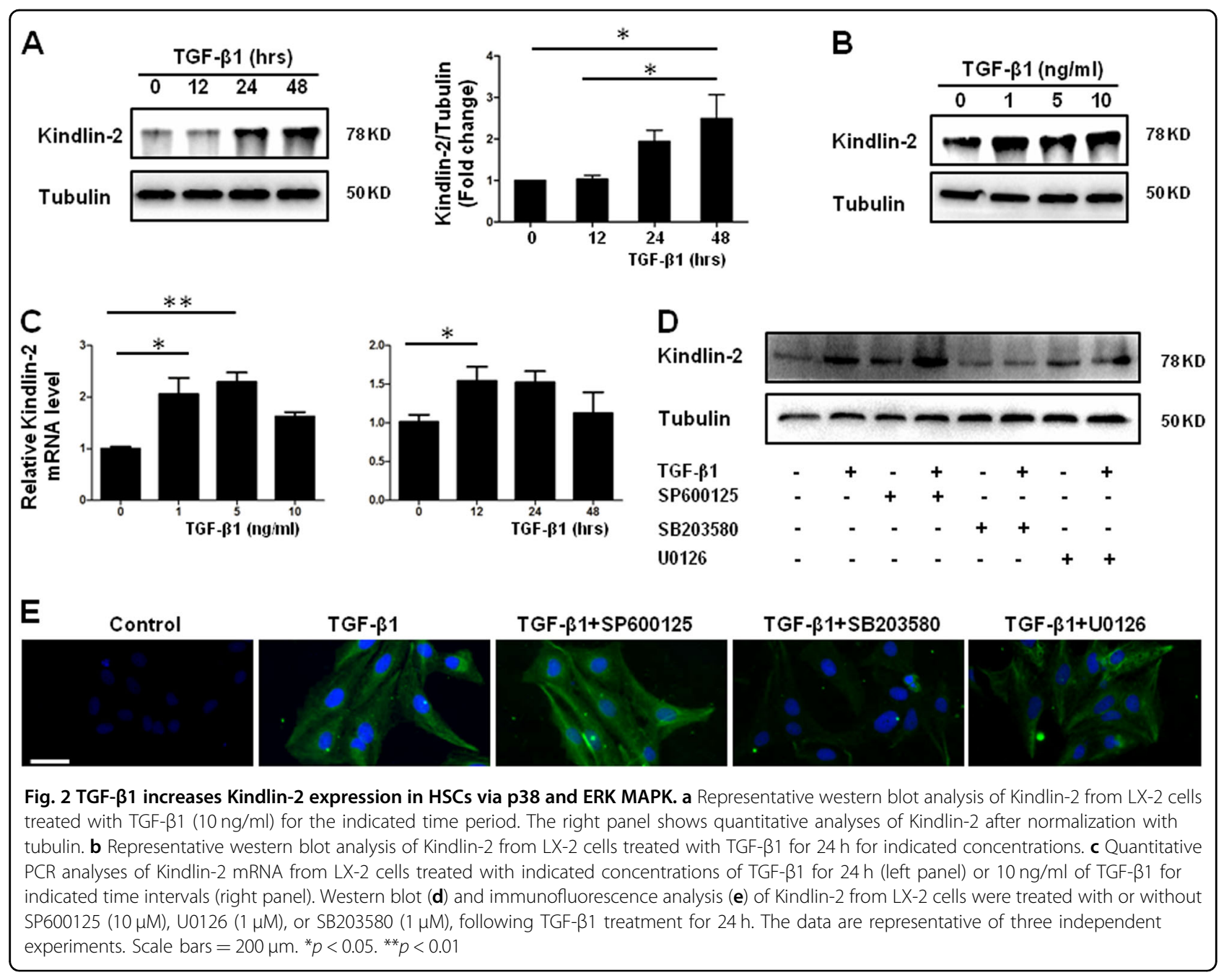

human liver fibrosis patients and mouse liver fibrosis model. Moreover, Kindlin-2 mRNA and protein levels were elevated in a time-dependent manner in TGF- $\beta 1$-stimulated LX-2 cells. This result was consistent with the recent reports that Kindlin-2 expression was increased in human fibrotic kidney and TGF- $\beta 1$-treated human renal tubular epithelial cells $(\mathrm{HKCs})^{21}$. In another study, Kindlin-2 was also increased significantly in pancreatic ductal adenocarcinoma (PDAC) cells stimulated with TGF- $\beta 1^{27}$. Nevertheless, little is known about the regulatory mechanism of increased Kindlin-2 expression induced by TGF- $\beta 1$. In the current study, TGF- $\beta 1$-induced Kindlin- 2 expression was abrogated by p38 and ERK inhibitor, which indicated that Kindlin-2 expression may be regulated, at least in part, by noncanonical Smad pathway. However, the detailed mechanism of how TGF- $\beta 1$ acts on the expression of Kindlin-2 needs further investigation.

HSC plays a pivotal role in the pathogenesis of liver fibrosis, and inhibition of HSC activation has been accepted as a major strategy to resolve liver fibrosis ${ }^{28,29}$.
Accumulating studies uncovered various pro-fibrogenic pathways involved in liver fibrosis, including TGF- $\beta$ / Smad, Wnt $/ \beta$-catenin, and PDGF pathways ${ }^{30}$. Among them, TGF- $\beta /$ Smad pathway is the most important in the progression of fibrotic diseases. Our study clearly demonstrated that knockdown of Kindlin-2 by siRNA dramatically reduced the TGF- $\beta 1$-induced HSC activation. Furthermore, siRNA-mediated silencing of Kindlin-2 inhibited TGF- $\beta 1$-induced $S \operatorname{mad} 2 / 3$ phosphorylation. These data suggest that Kindlin-2 is required for TGF- $\beta 1$ mediated HSC activation. However, more experiments should be carried out to unravel the precise mechanism.

Several independent teams have reported paradoxical results about the role of Kindlin-2 in fibrotic diseases. Wei et al. demonstrated that blockade of Kindlin-2 in vivo ameliorated unilateral ureteral obstruction (UUO)induced renal fibrosis ${ }^{21}$; on the contrary, postnatal loss of Kindlin-2 led to the enlargement of the heart and extensive fibrosis ${ }^{22,23}$. In this study, we clearly revealed that Kindlin-2 ${ }^{+/-}$mice were protected against $\mathrm{CCl}_{4}$-induced 


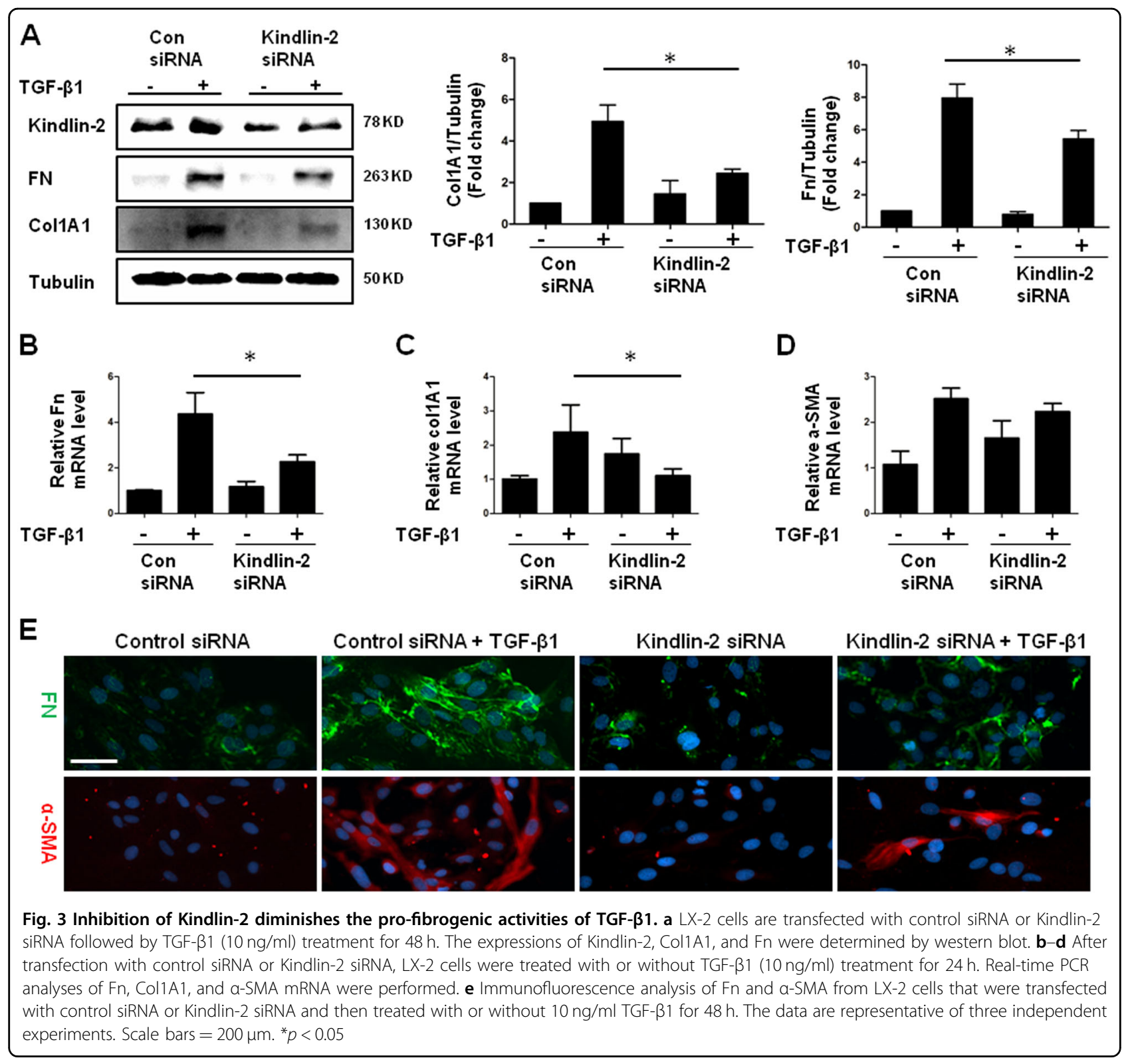

liver fibrosis, which is in line with our data of in vitro experiments. It is worth to note that we and other teams used different transgenic mice and fibrotic animal models. These findings indicate a distinct role of Kindlin-2 in different organ fibrogenesis. Thus, our study and theirs together provide strong evidence that Kindlin-2 plays a crucial role in fibrotic disorders, positive or negative. It will be interesting to evaluate the role of Kindlin-2 in other fibrotic diseases.

Liver fibrosis is a complex disorder that involves many cell types including HSC, hepatocytes, Kupffer cells, and infiltrated inflammatory cells ${ }^{4}$. Apart from activated HSC, hepatocytes derived from epithelial mesenchymal transition (EMT) have been revealed to be another important source of $\mathrm{ECM}^{31}$. Anyway, this study focuses on the effect of Kindlin-2 on HSC activation. It will be interesting to see if Kindlin-2 influences hepatocytes apoptosis and EMT during liver fibrosis. Although TGF$\beta 1$ is the most important pro-fibrogenic factor, accumulating studies have revealed multiple factors such as PDGF and Wnt are involved in the activation of HSCs and the pathogenesis of liver fibrosis ${ }^{32,33}$. Kindlin-2 acts as an adapter protein which forms protein complex with signaling components, then facilitates downstream signal transduction. Therefore, Kindlin-2 may likely participate in various intracellular signaling. In this study, we uncovered the role Kindlin- 2 in TGF- $\beta 1$ signaling; however, future studies should focus on the role of Kindlin-2 


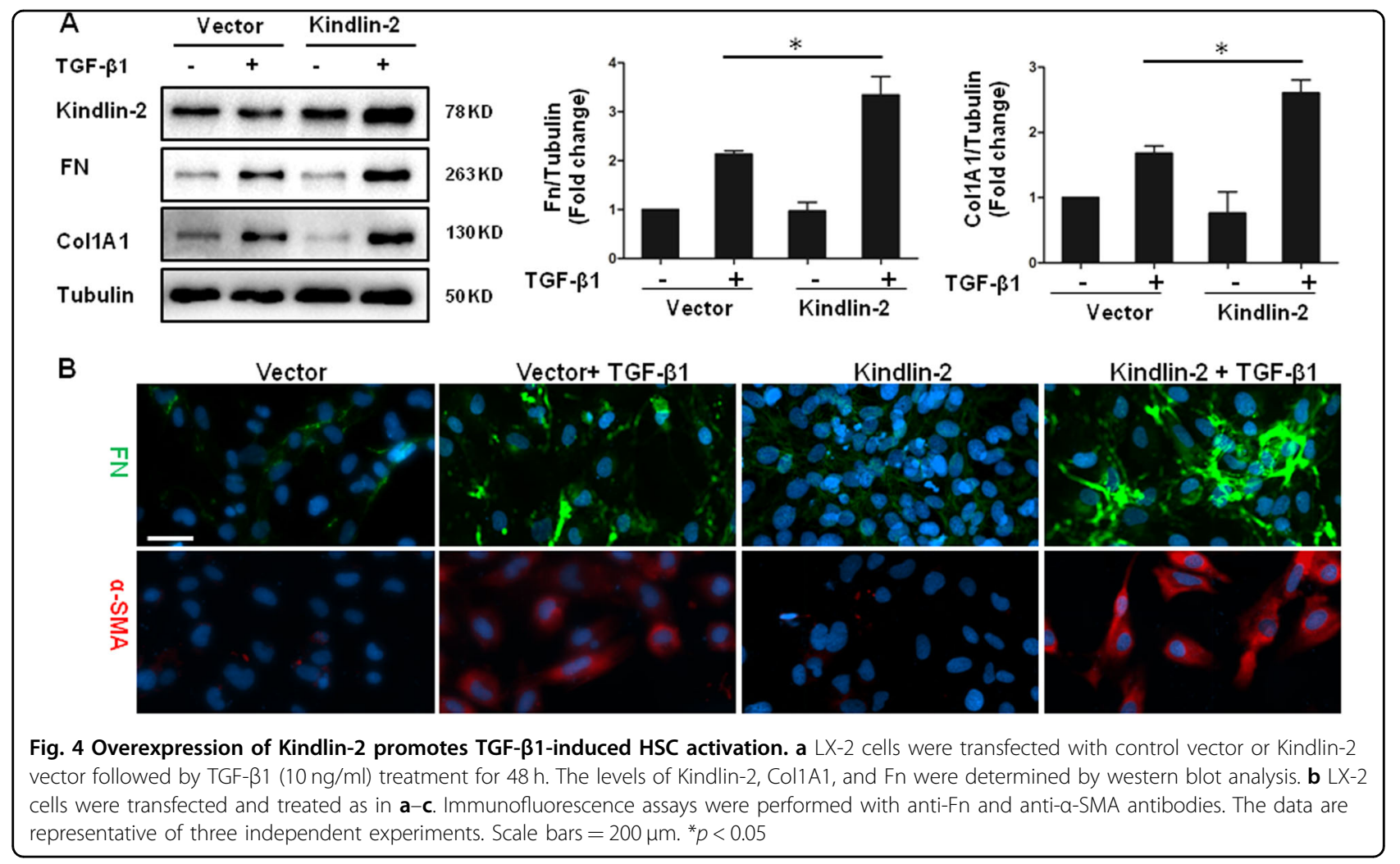

in other pro-fibrogenic signaling and the cross talk between multiple pathways.

In summary, our findings in the present study identified that Kindlin-2 may play a pivotal role in HSC activation and liver fibrosis progression by both in vitro and in vivo approaches. Kindlin- 2 is regulated by TGF- $\beta / \mathrm{Smad}$ pathway and contributes to the phosphorylation of Smad2 and Smad3. Importantly, Kindlin-2 depletion attenuates liver fibrosis in a $\mathrm{CCl}_{4}$ mouse model of liver fibrosis. These results indicate that Kindlin-2 plays a key role in the process of liver fibrogenesis and represent a new therapeutic target to effectively limit the progression of liver fibrosis.

\section{Materials and methods \\ Human liver samples}

Human liver samples were obtained from patients undergoing liver resection at the Tongji Hospital. The study protocol was approved by the Institutional Review Board of Tongji Hospital, and informed consent in writing was obtained from each patient.

\section{Mice}

Kindlin- $2^{+/-}$mice in the C57BL/6 background were purchased from the Model Animal Research Center of Nanjing University (Nanjing, China). Protocols for animal use were approved by the Institutional Animal Care and
Use Committee (IACUC) at Tongji Hospital in accordance with the National Institutes of Health guidelines. Liver fibrosis was induced by intraperitoneal injection of carbon tetrachloride $\mathrm{CCl}_{4}$ (1:5 dilution in olive oil, $1 \mathrm{ml} /$ $\mathrm{kg}$ body, three times per week for 6 weeks) ${ }^{34}$.

\section{Cell culture and treatment}

Human HSC cell line, LX-2 cells, was cultured in Dulbecco's modified Eagle's medium (DMEM) containing $10 \%$ fetal bovine serum (FBS) and 1\% penicillin/streptomycin, and maintained in a humidified incubator with $5 \%$ $\mathrm{CO}_{2}$ at $37^{\circ} \mathrm{C}$. LX-2 cells were serum-starved with $0.5 \%$ FBS for $24 \mathrm{~h}$ before intervention.

\section{Reagents and chemicals}

Recombinant human TGF- $\beta 1$ was purchased from Peprotech (Rocky Hill, NJ, USA), reconstituted in DMEM containing $10 \% \mathrm{FBS}$, and utilized at a final concentration of $10 \mathrm{ng} / \mathrm{ml}$. JNK inhibitor SP600125, ERK inhibitor U0126, and p38 MAPK inhibitor SB203580 were purchased from SelleckChem (Houston, TX, USA), dissolved in DMSO, and utilized at a final concentration of $10 \mu \mathrm{M}$, $1 \mu \mathrm{M}$, and $1 \mu \mathrm{M}$, respectively.

\section{RNA interference analysis}

siRNA targeting the Kindlin-2 sequences and nontargeting siRNA were synthesized by RiboBio Co., Ltd. 


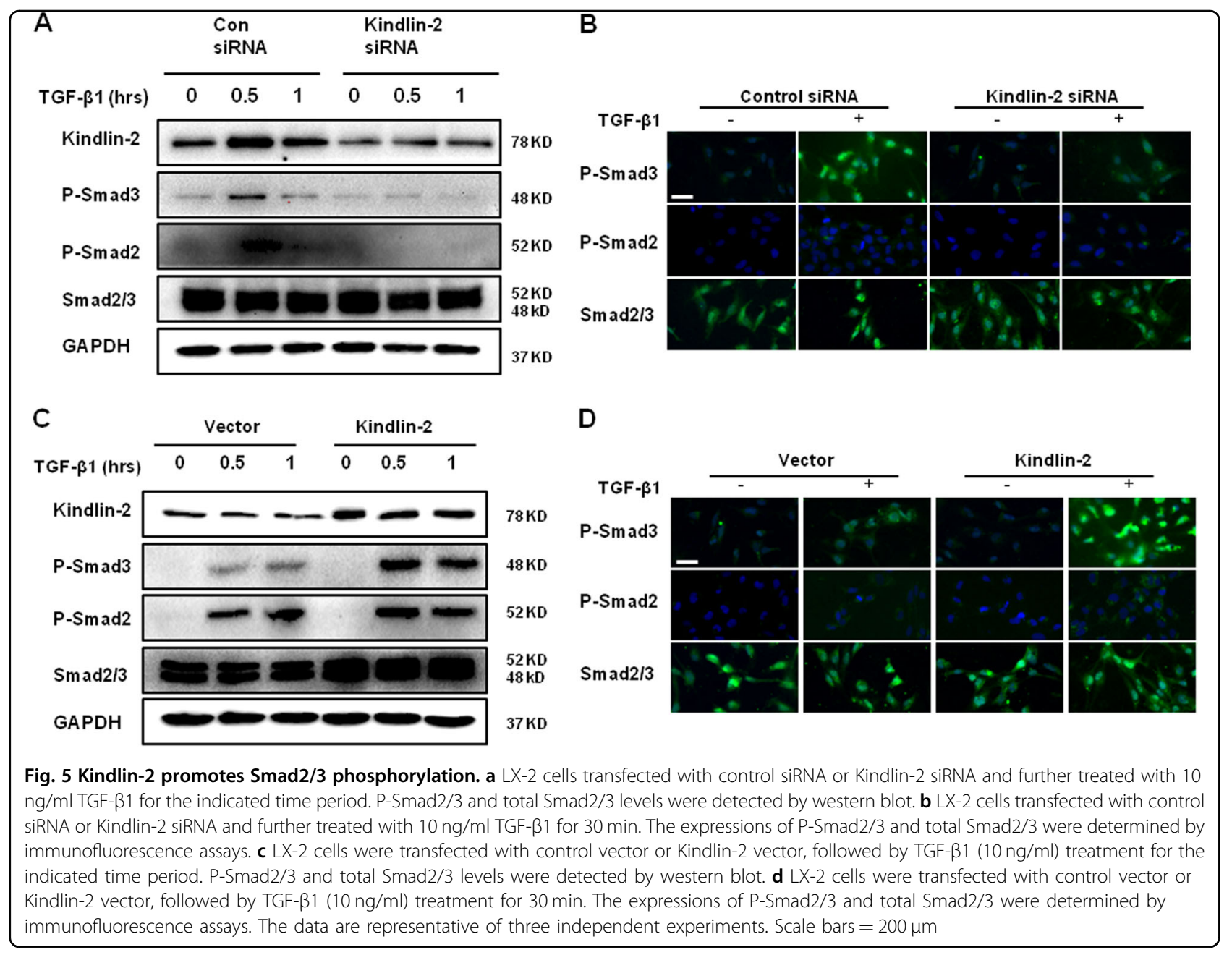

(Guangzhou, China). The sense targeting sequence was as follows: AAGCUGGUGGAGAAACUCG ${ }^{17}$. LX-2 cells were transfected with Kindlin-2 or control siRNA at 50\% confluence using Lipofectamine 2000 (Invitrogen) according to the manufacturer's instructions.

\section{Overexpression of Kindlin-2}

To force overexpression of Kindlin-2, pEGFP-Kindlin-2 (Vigene Biosciences, Shandong, China) was transfected into LX-2 cells using Lipofectamine 2000. Twenty-four hours after transfection, the cells were treated as indicated and assessed by western blot.

\section{Western blot analysis}

Western blot analysis was performed as described previously $^{35}$. The primary antibodies used in western blot were: Kindlin-2 (Proteintech, 1:1000 dilution), Fibronectin (FN) (Proteintech, 1:1000 dilution), Col1A1 (Boster, 1:400 dilution), $\alpha$-SMA (Abcam, 1:100 dilution), Smad2/3 (Cell Signaling Technology, 1:1000 dilution), p-Smad2 (Cell
Signaling Technology, 1:1000 dilution), p-Smad3 (Cell Signaling Technology, 1:1000 dilution), GAPDH (Abacm, 1:3000 dilution), $\beta$-actin (Abacm, 1:3000 dilution), and tubulin (Abcam, 1:3000 dilution). Detection was performed using a chemiluminescent substrate system (BioRad). The gray values were analyzed with ImageJ software.

\section{Quantitative real-time polymerase chain reaction}

Total RNA from liver tissues or LX-2 cells was extracted by Trizol (Takara, Dalian, China). The single-stranded cDNA was synthesized from total RNA using PrimeScript ${ }^{\mathrm{TM}}$ RT reagent kit (Takara). Real-time PCR was set up using SYBR Green mix (Takara) under the following condition: $30 \mathrm{~s}$ at $95^{\circ} \mathrm{C}$ for initial denaturation, followed by 40 cycles of $95^{\circ} \mathrm{C}$ for $5 \mathrm{~s}$ and $60^{\circ} \mathrm{C}$ for $30 \mathrm{~s}$ on the Applied Biosystems 7500 Real-Time PCR system (Thermo Fisher Scientific, USA). The levels of $\beta$-actin RNA expression were used to normalize the data. The sequences of primers for real-time PCR are listed in Supplementary Table 1. 


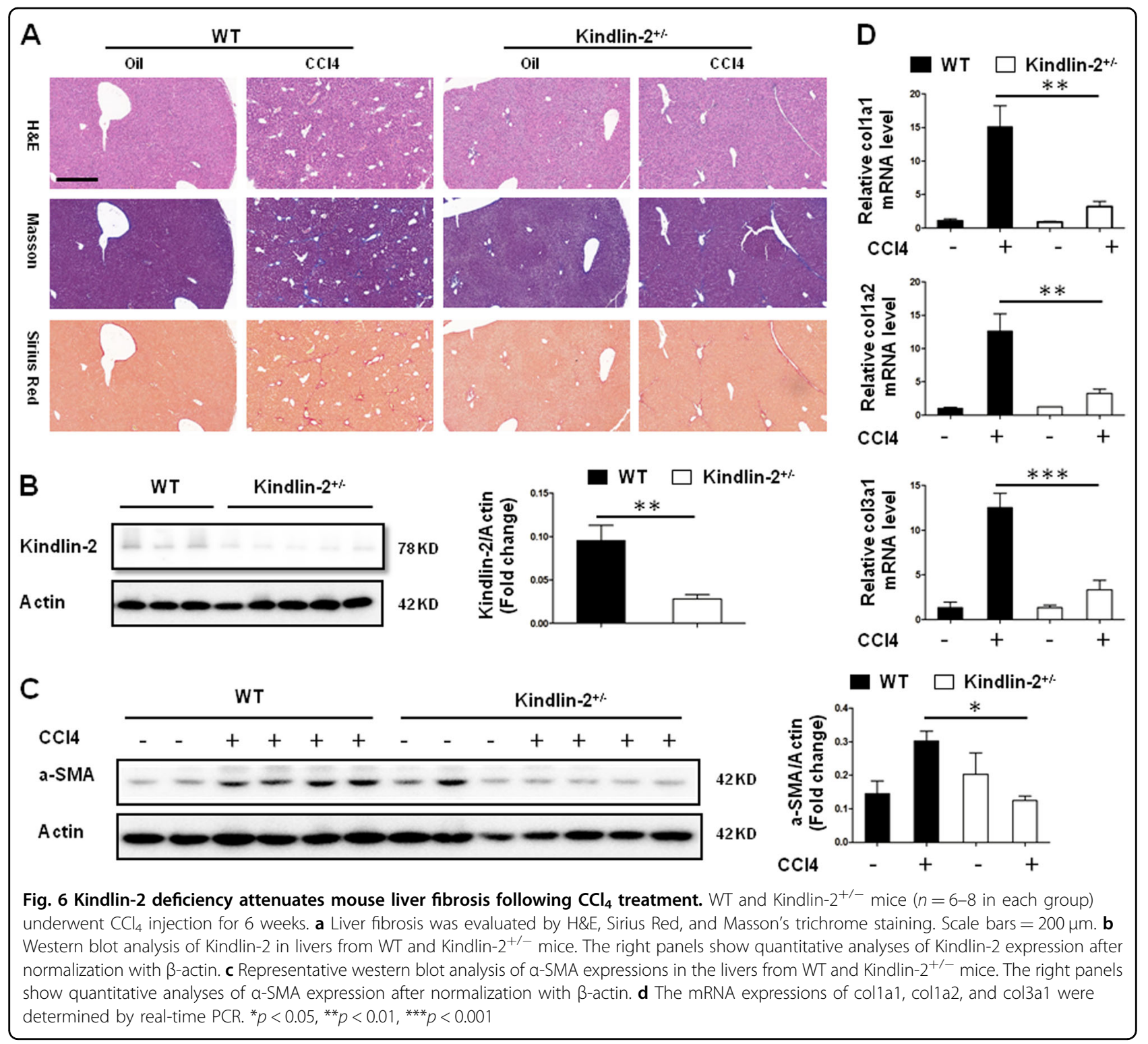

\section{Histological analysis of liver sections}

The specimens were fixed in $4 \%$ paraformalin and embedded in paraffin in preparation for histopathological analysis. Sections were stained with hematoxylin and eosin (H\&E) for morphology, and Masson's trichrome and Sirius red for fibrosis.

\section{Immunofluorescence staining}

Immunofluorescence staining was performed as described previously ${ }^{36}$. Briefly, cells cultured on the glass coverslips or tissue sections were stained with following antibodies: Kindlin-2 (Proteintech, 1:100 dilution), Fibronectin (FN) (Abcam, 1:100 dilution), $\alpha$-SMA (Abcam, 1:100 dilution), Smad2/3 (Cell Signaling Technology, 1:100 dilution), p-Smad2 (Cell
Signaling Technology, 1:100 dilution), and p-Smad3 (Cell Signaling Technology, 1:100 dilution) overnight at $4{ }^{\circ} \mathrm{C}$, followed by incubation with FITC or PEconjugated secondary antibodies (Invitrogen). The nuclei were the stained with DAPI. All immunofluorescence was then visualized by Carl Zeiss MicroImaging system (Carl Zeiss, Germany).

\section{Statistical analysis}

Data were expressed as mean \pm SEM. Differences between two groups were examined for statistical significance using the Student's $t$ test. For comparisons between multiple groups, one-way ANOVA followed by Tukey's test was performed. $P<0.05$ was considered significant. 


\section{Funding}

This work was supported by grants from the National Natural Science Foundation of China (81600472).

\begin{abstract}
Author details
'Department of Thoracic Surgery, Tongji Hospital of Tongji Medical College, Huazhong University of Science and Technology, Wuhan, China. ${ }^{2}$ Department of Respiratory and Critical Care Medicine, Tongji Hospital, Tongji Medical College, Huazhong University of Science and Technology, Wuhan, China. ${ }^{3}$ Hepatobiliary and Pancreas Diagnosis and Treatment Center, Shiyan Taihe Hospital, Hubei University of Medicine, Shiyan, Hubei, China. ${ }^{4}$ Department of Infectious Disease, Tongji Hospital of Tongji Medical College, Huazhong University of Science and Technology, Wuhan, China
\end{abstract}

\section{Conflict of interest}

The authors declare that they have no conflict of interest.

\section{Publisher's note}

Springer Nature remains neutral with regard to jurisdictional claims in published maps and institutional affiliations.

The online version of this article (https://doi.org/10.1038/s41420-018-0095-9) contains supplementary material, which is available to authorized users.

Received: 9 January 2018 Revised: 13 February 2018 Accepted: 5 August 2018

Published online: 12 September 2018

\section{References}

1. Pellicoro, A., Ramachandran, P., Iredale, J. P. \& Fallowfield, J. A. Liver fibrosis and repair: immune regulation of wound healing in a solid organ. Nat. Rev. Immunol. 14, 181-194 (2014).

2. Hernandez-Gea, V. \& Friedman, S. L. Pathogenesis of liver fibrosis. Annu. Rev. Pathol. 6, 425-456 (2011).

3. Kisseleva, T. The origin of fibrogenic myofibroblasts in fibrotic liver. Hepatology 65, 1039-1043 (2017)

4. Bataller, R. \& Brenner, D. A. Liver fibrosis. J. Clin. Invest. 115, 209-218 (2005).

5. Biernacka, A., Dobaczewski, M. \& Frangogiannis, N. G. TGF-beta signaling in fibrosis. Growth Factors 29, 196-202 (2011).

6. Akhurst, R. J. \& Hata, A. Targeting the TGFbeta signalling pathway in disease. Nat. Rev. Drug Discov. 11, 790-811 (2012).

7. Zhang, Y. E. Non-Smad pathways in TGF-beta signaling. Cell Res. 19, 128-139 (2009).

8. Xu, F., Liu, C., Zhou, D. \& Zhang, L. TGF-beta/SMAD pathway and its regulation in hepatic fibrosis. J. Histochem. Cytochem. 64, 157-167 (2016).

9. Calderwood, D. A., Campbell, I. D. \& Critchley, D. R. Talins and kindlins: partners in integrin-mediated adhesion. Nat. Rev. Mol. Cell Biol. 14, 503-517 (2013).

10. Larjava, H., Plow, E. F. \& Wu, C. Kindlins: essential regulators of integrin signalling and cell-matrix adhesion. EMBO Rep. 9, 1203-1208 (2008).

11. Rognoni, E., Ruppert, R. \& Fassler, R. The kindlin family: functions, signaling properties and implications for human disease. J. Cell. Sci. 129, 17-27 (2016).

12. Montanez, E. et al. Kindlin-2 controls bidirectional signaling of integrins. Genes Dev. 22, 1325-1330 (2008).
13. Hirbawi, J. et al. The extreme C-terminal region of kindlin-2 is critical to its regulation of integrin activation. J. Biol. Chem. 292, 14258-14269 (2017).

14. Ma, Y. Q., Qin, J., Wu, C. \& Plow, E. F. Kindlin-2 (Mig-2): a co-activator of beta3 integrins. J. Cell Biol. 181, 439-446 (2008).

15. Talaat, S. et al. Kindlin-2 expression in arsenite- and cadmium-transformed bladder cancer cell lines and in archival specimens of human bladder cancer. Urology 77, 1507.e1501-1507 (2011).

16. Shen, Z. et al. Kindlin-2: a novel adhesion protein related to tumor invasion, lymph node metastasis, and patient outcome in gastric cancer. Am. J. Surg. 203, 222-229 (2012).

17. An, Z. et al. Kindlin-2 is expressed in malignant mesothelioma and is required for tumor cell adhesion and migration. Int. J. Cancer 127, 1999-2008 (2010).

18. Jung, G. Y., Park, Y. J. \& Han, J. S. Mediation of Rac1 activation by kindlin-2: an essential function in osteoblast adhesion, spreading, and proliferation. J. Cell. Biochem. 112, 2541-2548 (2011).

19. Gong, X. et al. Kindlin-2 controls sensitivity of prostate cancer cells to cisplatininduced cell death. Cancer Lett. 299, 54-62 (2010).

20. Moslem, M. et al. Kindlin-2 modulates the survival, differentiation, and migration of induced pluripotent cell-derived mesenchymal stromal. Cells 2017, 7316354 (2017)

21. Wei, X. et al. Kindlin-2 mediates activation of TGF-beta/Smad signaling and renal fibrosis. J. Am. Soc. Nephrol. 24, 1387-1398 (2013).

22. Qi, L. et al. Depletion of Kindlin-2 induces cardiac dysfunction in mice. Sci. China Life Sci. 59, 1123-1130 (2016).

23. Zhang, Z. et al. Postnatal loss of Kindlin-2 leads to progressive heart failure. Circ. Heart Fail. 9, e003129 (2016).

24. Liu, Y. et al. Animal models of chronic liver diseases. Am. J. Physiol. Gastrointest. Liver Physiol. 304, G449-G468 (2013).

25. $\mathrm{Xu}$, L. et al. Human hepatic stellate cell lines, $L X-1$ and $L X-2$ : new tools for analysis of hepatic fibrosis. Gut 54, 142-151 (2005).

26. Lin, J. et al. Kindlin-2 promotes hepatocellular carcinoma invasion and metastasis by increasing Wnt/beta-catenin signaling. J. Exp. Clin. Cancer Res. 36, 134 (2017).

27. Zhan, J. et al. Kindlin-2 induced by TGF-beta signaling promotes pancreatic ductal adenocarcinoma progression through downregulation of transcriptional factor HOXB9. Cancer Lett. 361, 75-85 (2015).

28. Lee, U. E. \& Friedman, S. L. Mechanisms of hepatic fibrogenesis. Best Pract. Res. Clin. Gastroenterol. 25, 195-206 (2011).

29. Friedman, S. L. Mechanisms of hepatic fibrogenesis. Gastroenterology 134 1655-1669 (2008)

30. Seki, E. \& Schwabe, R. F. Hepatic inflammation and fibrosis: functional links and key pathways. Hepatology 61, 1066-1079 (2015).

31. Popov, Y. \& Schuppan, D. Targeting liver fibrosis: strategies for development and validation of antifibrotic therapies. Hepatology $\mathbf{5 0}$, 1294-1306 (2009).

32. Miao, C. G. et al. Wht signaling in liver fibrosis: progress, challenges and potential directions. Biochimie 95, 2326-2335 (2013).

33. Borkham-Kamphorst, E. \& Weiskirchen, R. The PDGF system and its antagonists in liver fibrosis. Cytokine Growth Factor Rev. 28, 53-61 (2016).

34. Lei, X. F. et al. Hic-5 deficiency attenuates the activation of hepatic stellate cells and liver fibrosis through upregulation of Smad7 in mice. J. Hepatol. 64, 110-117 (2016).

35. Zeng, Z. et al. Activation and overexpression of Sirt1 attenuates lung fibrosis via P300. Biochem. Biophys. Res. Commun. 486, 1021-1026 (2017).

36. Chen, H. et al. CXCR4 inhibitor attenuates allergen-induced lung inflammation by down-regulating MMP-9 and ERK1/2. Int. J. Clin. Exp. Pathol. 8, 6700-6707 (2015). 